\title{
Quantitative Scintigraphy with Deconvolutional Analysis for the Dynamic Measurement of Hepatic Function
}

\author{
Edward P. TagGe, M.D., Darrell A. Campbell, JR., M.D., ${ }^{1}$ RalPh Reichle, \\ DAMON R. AVERILL, JR., D.V.M., ROBERT M. MERION, M.D., \\ Donald C. Dafoe, M.D., Jeremiah G. Turcotte, M.D., And JaCk E. Juni, M.D. \\ Departments of Surgery and Internal Medicine, The University of Michigan \\ Medical Center, Ann Arbor, Michigan 48104
}

Presented at the Annual Meeting of the Association for Academic Surgery, Washington, D.C., November 5-8, 1986

\begin{abstract}
A mathematical technique known as deconvolutional analysis was used to provide a critical and previously missing element in the computations required to quantitate hepatic function scintigraphically. This computer-assisted technique allowed for the determination of the time required, in minutes, of a labeled bilirubin analog ( ${ }^{99 \mathrm{~m}} \mathrm{Tc}$-disofenin) to enter the liver via blood and exit via bile. This interval was referred to as the mean transit time (MTT). The critical process provided for by deconvolution is the mathematical simulation of a bolus injection of tracer directly into the afferent blood supply of the liver. The raw data required for this simulation are obtained from the intravenous injection of labeled disofenin, a member of the HIDA family of radiopharmaceuticals. In this study, we perform experiments which document that the simulation process itself is accurate. We then calculate the MTT under a variety of experimental conditions involving progressive hepatic ischemia/reperfusion injury and correlate these results with the results of simultaneously performed BSP determinations and hepatic histology. The experimental group with the most pronounced histologic findings (necrosis, vacuolization, disorganization of hepatic cords) also have the most prolonged MTT and BSP half-life. However, both quantitative imaging and BSP testing are able to identify milder degrees of hepatic ischemic injury not reflected in the histologic evaluation. Quantitative imaging with deconvolutional analysis is a technique easily adaptable to the standard nuclear medicine minicomputer. It provides rapid results and appears to be a sensitive monitor of hepatic functional disturbances resulting from ischemia and reperfusion. (c) 1987 Academic Press, Inc.
\end{abstract}

\section{INTRODUCTION}

There is currently no reliable method to assess acute changes in liver function. One must rely on clinical findings or measure changes in the level of substances metabolized by the liver, a process which involves a considerable time delay between cause and effect. In this report we describe a scintigraphic technique coupled with a computer-assisted mathematical interpretation which reliably assesses hepatic function on an acute basis. Because this test is sensitive, minimally invasive, and very rapid, it has great potential for clinical application. In particular, this

\footnotetext{
${ }^{1}$ To whom correspondence and reprint requests should be addrcssed: Department of Surgery, University of Michigan Medical Center, 2926D Taubman Center, Box 0331, Ann Arbor, MI 48109.
}

technique lends itself to use in the context of liver transplantation, where rapid changes in the function of the newly transplanted, or potentially rejecting, liver are of paramount importance.

The scintigraphic measurement of hepatic function requires the following: a test substance must be handled exclusively by the liver; it must be secreted into the bile without significant "leak" back into the blood stream; and it must be presented to the liver in the form of a single, short intravascular bolus. If these conditions are met, it is a straightforward matter to measure the total uptake of the test substance by the liver and to quantitate subsequent excretion. The introduction of the HIDA family of scintigraphic reagents in 1975 [1, 2] provided drugs capable of satisfying the first two crite- 
ria $[3,4]$ but it has not been possible in the clinical setting to administer the test substance in question directly into the dual blood supply of the liver, the portal vein, and hepatic artery. The intravenous injection of test substance is not an adequate substitute since the liver receives only a fraction of the total cardiac output, and substantial amounts of the injected material would bypass the liver on the first pass, to be "recirculated" through the liver subsequently. Because of recirculation, the liver is presented with a continually changing concentration of tracer. This, coupled with simultaneous extraction of tracer from blood by the liver, makes it impossible to define a baseline initial level of maximum uptake and to precisely measure the subsequent rate of excretion.

In this paper we use a technique called deconvolution, which analyzes data obtained from the intravenous injection of ${ }^{99 \mathrm{~m}}$ Tc-disofenin and mathematically constructs a simulated bolus injection of tracer into the blood supply of the liver. In this simulated injection, tracer is presented to the liver initially, but the effects of recirculation are corrected so that measurement of the time required for transit of tracer through and out of the liver is easily calculated.

\section{MATERIAL AND METHODS}

\section{${ }^{99 m}$ Tc-Disofenin Scan with Deconvolution}

In order to quantitatively measure hepatocyte function in the acute setting, we performed scintigraphic imaging with ${ }^{99 \mathrm{~m}} \mathrm{Tc}$ disofenin, a member of the HIDA family of tracers. Imaging was initiated within $30 \mathrm{~min}$ of completion of the surgical procedure. Each animal was imaged using a wide fieldof-view gamma camera positioned ventrally (General Electric 400 AT camera with general-purpose low-energy parallel hole collimator) so as to include both the heart and liver in the field. Each animal was then injected intravenously with $5 \mathrm{mCi}{ }^{99 m}$ Tc-disofenin and continuous 1 -min scintigraphic images were acquired for $32 \mathrm{~min}$. These images were stored directly on a commercial nuclear medicine minicomputer (Gencral Electric Star minicomputer).

After data acquisition, computerized regions-of-interest of the heart and liver were drawn on the images. Using these, we generated plots of the changing amount of radioactivity in the heart and liver over time (time-activity curves). Since there is no cardiac uptake of the tracer, the plot of radioactivity in the heart is analogous to a plot of the changing blood concentration of tracer over time. To correct the liver time-activity curve for the effects of continuing systemic recirculation of tracer and the constantly changing blood concentration of tracer, the computer was utilized to perform deconvolution of the liver curves. This was done by employing the blood (heart) curve as a representative input function using a computer program previously developed at this institution [5]. This method has been validated by computer simulation as well as in vivo models [6] and has proven useful in differentiating intrahepatic from extrahepatic jaundice [7]. This procedure results in a liver time-activity curve which has been "corrected" for the effects of systemic recirculation of tracer. The hepatic mean transit time (MTT) of tracer was then analyzed by integrating the area under this curve and dividing by the maximum height of the curve.

The initial points of the "corrected" or deconvolved hepatic time-activity curve represent the total tracer activity presented to the liver. Later points in the curve represent the relative amount of the initial tracer administered that is retained and excreted by the liver.

\section{Direct Administration of ${ }^{99 m} \mathrm{Tc}$-Disofenin into the Afferent Blood Supply of the Liver}

To validate these measurements, we compared the liver function of a normal pig liver assessed using the intravenous administration of ${ }^{99 \mathrm{~m}} \mathrm{Tc}$-disofenin and deconvolution 
(indirect method) to results obtained when the same agent was simultaneously administered directly into both the hepatic artery and the portal vein (direct method). For the latter technique, pigs were operated upon through a midline incision and the gastroduodenal artery and gastroduodenal vein were cannulated with a small piece of polyethylene tubing connected to a syringe and three-way stopcock. The right gastric artery and any smaller branches leading to the stomach were ligated and divided so that the entire amount of injected radionuclide reached the liver. Five millicuries of ${ }^{99 \mathrm{~m}} \mathrm{Tc}$ disofenin for injection was then divided so that two-thirds was administered via the portal vein and one-third was administered via the hepatic artery. This somewhat arbitrary division was based on the normal 2:1 ratio of portal vein to hepatic arterial blood flow observed in humans [8]. Computer image acquisition was started and both doses were injected simultaneously into the portal vein and hepatic artery and were followed with a 3 -cc flush of heparinized normal saline. After data acquisition, the indirect assay was performed in the same animal so that each served as its own control.

\section{BSP Retention}

The clearance rate of bromosulfophthalein (BSP) is a commonly used indicator of liver function in domestic animals [9]. The presence of liver dysfunction increases the concentration of BSP at a specified time and thus prolongs plasma half-life.

Sterile disodium phenoltetrabromophthalein disulfanate $(5 \mathrm{mg} / \mathrm{kg})$ was administered intravenously $\mathrm{I} \mathrm{hr}$ following reperfusion of the liver with blood. Blood samples were collected at 5 and $30 \mathrm{~min}$. A spectrophotometric determination at $565 \mathrm{mu}$ was performed following the addition of $1.0 \mathrm{~N} \mathrm{NaOH}$ and distilled water to each plasma sample. The concentration of BSP was then derived from a previously constructed standard curve.
Half-lives were calculated using the following formula:

$$
T_{1 / 2}=\frac{0.693}{2.303\left(\left(\log C_{1}-\log C_{2}\right) /\left(T_{2}-T_{1}\right)\right)}
$$

$T_{1}=5 \mathrm{~min}$

$T_{2}=30 \mathrm{~min}$

$C_{1}=[\mathrm{BSP}]$ at $5 \mathrm{~min}$

$C_{2}=[\mathrm{BSP}]$ at $30 \mathrm{~min}$

\section{Transplantation Technique}

The technique for porcine allograft liver transplantation has been described elsewhere $[10,11]$. Briefly, donor livers were harvested after in situ perfusion of the portal vein with cold $\left(4^{\circ} \mathrm{C}\right)$ Eurocollins solution. While $e x$ vivo, a total of $1500 \mathrm{ml}$ of cold perfusate was administered through the portal vein and hepatic artery. The donor livers were subjected to an average cold ischemic time of $1 \mathrm{hr}$.

Recipient hepatectomy was performed following placement of a passive veno-venous bypass circuit. Blood from both the portal vein and the infrahepatic vena cava was shunted via a Y-connector to the right external jugular vein. The suprahepatic vena caval anastomosis was performed first, followed by the portal vein. Prior to completion of the latter anastomosis, $1000 \mathrm{cc}$ of warm saline was perfused through the portal vein. Both this maneuver and the administration of intravenous calcium chloride were used to protect against hyperkalemia which occurs immediately upon revascularization (removal of the portal vein and suprahepatic clamps) [12]. The average warm ischemic time (out of ice) was $35 \mathrm{~min}$. The infrahepatic vena cava, hepatic artery (end to side to recipient common hepatic), and bile duct anastomoses were then completed in sequence.

\section{Ischemic Technique}

In order to further study the ${ }^{99 \mathrm{~m}} \mathrm{Tc}$-disofenin scan, an operative technique was de- 
vised to subject the liver to warm absolute ischemia. Initial hepatic dissection and placement of a veno-venous bypass circuit were carried out, similar to the transplant operation. However, instead of removing the liver, Satinsky clamps were placed across the remainder of the portal triad and around the suprahepatic vena cava, thus rendering the liver totally ischemic. After the prescribed ischemic length, the clamps and shunt were removed, and the portal vein and infrahepatic vena caval venotomies were closed with a running 5-O Tevdek. One hour following revascularization, BSP measurements were performed and the pigs were then scanned.

\section{Histologic Evaluation}

Open liver biopsies taken from the free edge of the left lobe were routinely obtained $1 \mathrm{hr}$ following revascularization and processed for histologic evaluation. Light microscopic evaluation was performed by one of us in blinded fashion. Slides were evaluated for various histologic changes, with necrosis, vacuolar changes, and disorganization of hepatic cords being the most discriminatory characteristics.

\section{RESULTS}

Figure 1 demonstrates the scintigraphic appearance of the liver after the direct (A) and indirect (B) injections of ${ }^{99 \mathrm{~m}} \mathrm{Tc}$-disofenin. After direct injection, only the liver is demonstrated and neither lung nor heart activity is observed, owing to the very high extraction efficiency of the liver for disofenin. In contrast, after an iv injection of ${ }^{99 \mathrm{~m}} \mathrm{Tc}$-disofenin both heart and liver are readily observed (B). Figure 2 shows the time-activity curves generated from the indirect and direct injections of ${ }^{99 \mathrm{~m}} \mathrm{Tc}$-disofenin for three normal animals. The calculated correlation coefficient was 0.95 . The calculated mean transit time MTT) for each technique in each animal is shown in Table 1. A paired $t$ test comparing MTT determinations after direct
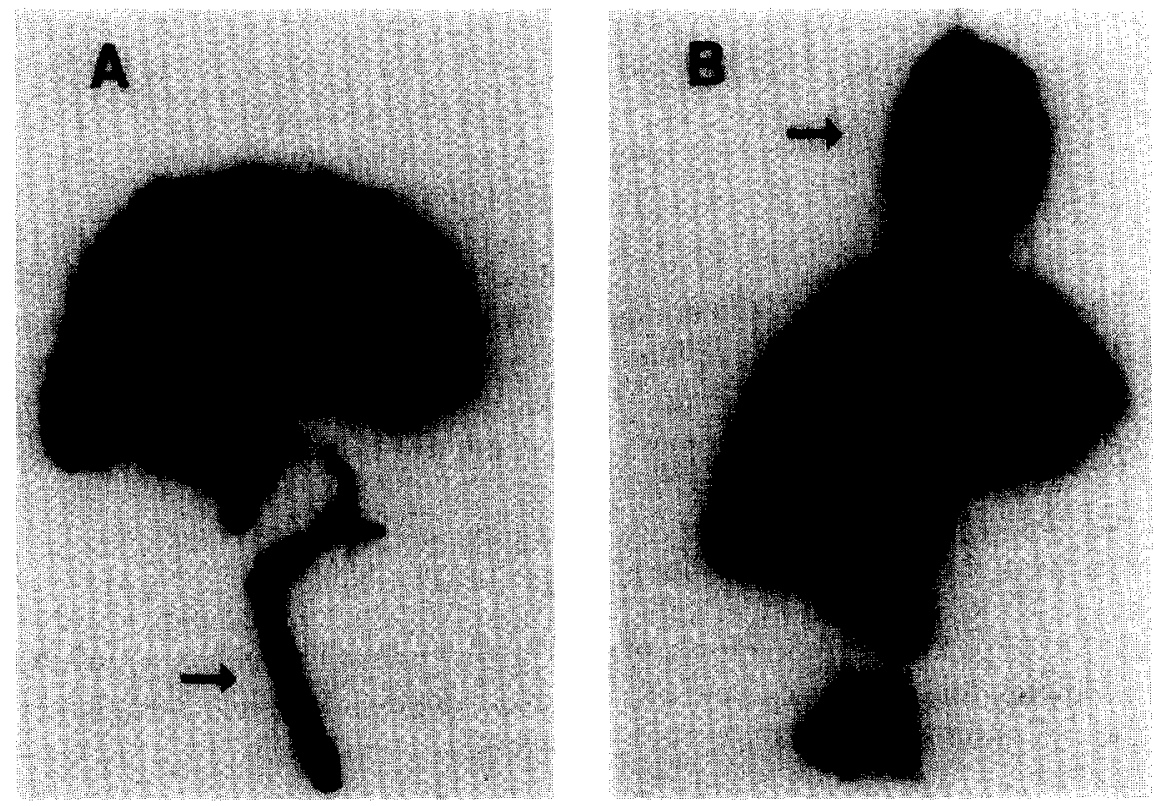

FIG. 1. Scintigraphic appearance after direct injection of ${ }^{99 \mathrm{~m}} \mathrm{Tc}$-disofenin into portal vein and hepatic artery (A) and after intravenous injection (B). In contrast to the intravenous injection, after direct injection no tracer is identified in the area of the heart. The arrow in (A) indicates the site of injection of tracer into the portal vein and hepatic artery. The arrow in (B) indicates tracer in the chambers of the heart. 


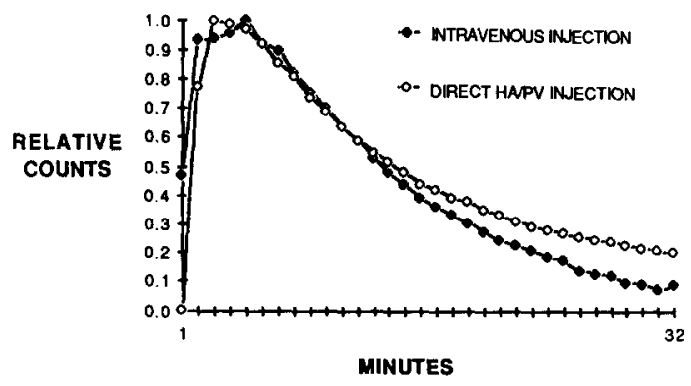

FIG. 2. Hepatic time-activity curves for the liver after intravenous injection of ${ }^{99 \mathrm{~m}} \mathrm{Tc}$-disofenin or direct injection of ${ }^{99 \mathrm{~m}} \mathrm{~T} \mathrm{c}$-disofenin into the portal vein and hepatic artery. The correlation coefficient for these curves was 0.95 .

and indirect measurements did not reveal a statistically significant difference.

One animal suffered an unexplained cardiac arrest during placement of the gastroduodenal artery and vein catheters and was asystolic for several minutes before resuscitation was begun. After successful resuscitation, which included open cardiac massage and the administration of epinephrine, the experiment proceeded, although the liver appeared to be distinctly abnormal (mottled) in appearance thereafter. However, the curves again appeared similar and the calculated MTTs were also similar although prolonged compared to the three normal animals (Table 1).

\section{Sensitivity of Quantitative Hepatic}

Scintigraphy and BSP Determinations to Varying Degrees of Ischemic Injury and

Correlation with Hepatic Histology

We considered it important to document the degree to which quantitative scintigraphy using the deconvolution technique could distinguish between varying degrees of hepatic ischemic injury. Pigs were subjected to 0.5 or $1.0 \mathrm{hr}$ of warm hepatic ischemia. In addition some pigs underwent orthotopic allotransplantation, which involved a $1-\mathrm{hr}$ period of cold ischemia (Collins preservation solution and ice storage) and an additional 35-min period of warm ischemia. After a 1-hr period of postischemia reperfusion for all experimental groups, BSP determinations were done, quantitative imaging was accomplished, and biopsies were taken.

The calculated half-life for BSP following injection was incrementally prolonged with increasing amounts of ischemic injury (Fig. 3 ) ranging from a $T_{1 / 2}$ of $43 \mathrm{~min}$ in the recipients of transplants stored in ice for $1 \mathrm{hr}$. Likewise the mean transit time (MTT) for ${ }^{99 \mathrm{~m}} \mathrm{Tc}$ disofenin obtained scintigraphically showed an increasingly prolonged transit of ${ }^{99 \mathrm{~m}} \mathrm{Tc}$ disofenin through the liver after warm ischemia and transplantation. Differences observed between normal and $0.5-\mathrm{hr}$ warm ischemia, 0.5- and 1.0-hr warm ischemia, and 1.0-hr warm ischemia and transplanted animals were statistically significant $(P$ $<0.05$ ) for both the scintigraphic technique and the BSP determinations.

Evaluation of liver biopsy specimens in the various experimental groups failed to show any reproducible pattern of injury for the animals subjected to 0.5 or $1.0 \mathrm{hr}$ of warm ischemia. Animals undergoing transplantation involving $1 \mathrm{hr}$ of cold storage were found to have substantial lobular necrosis, vacuolization, and hepatic cord disorganization when compared to the other groups (Table 2).

TABLE 1

MTT DETERMINATIONS CALCULATED FROM DIRECT ${ }^{a}$ AND INDIRECT ${ }^{b}$ INJECTIONS OF ${ }^{99 \mathrm{~m}}$ TC-DISOFENIN

\begin{tabular}{ccc} 
Experiment animal & Direct & Indirect \\
\hline 1 & 16.2 & 14.1 \\
2 & 13.7 & 13.9 \\
3 & 16.7 & 15.1 \\
$4^{c}$ & 21.6 & 19.8 \\
\hline
\end{tabular}

${ }^{a}$ Direct simultaneous injections of ${ }^{99} \mathrm{~m}$ Tc-disofenin into portal vein and hepatic artery in a ratio of $2: 1$.

${ }^{b}$ Indirect refers to the intravenous injection of ${ }^{99 \mathrm{~m}} \mathrm{Tc}-$ disofenin and subsequent calculation of MTT using deconvolution.

${ }^{c}$ This animal suffered a cardiac arrest prior to the initiation of this scan. 


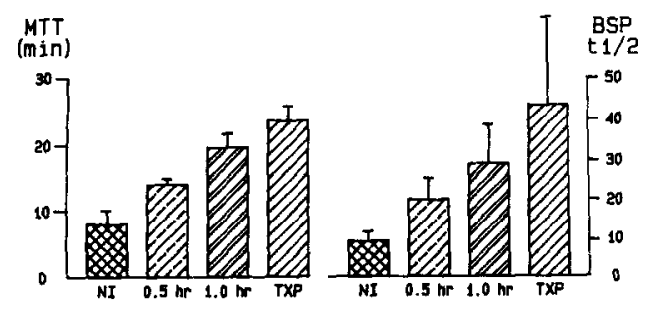

FIG. 3. Sensitivity of quantitative scintigraphy and BSP testing to various degrees of hepatic ischemia/reperfusion injury. (Left) MTT determinations were done for several different groups; normal $(n=6)$, animals subjected to $0.5-\mathrm{hr}$ warm ischemia $(n=4)$ or $1.0 \mathrm{hr}$ warm ischemia ( $n=4$ ), or animals undergoing orthotopic transplantation $(n=6)$. Statistically significant differences were noted between normal and 0.5 -hr warm ischemia, 0.5- and 1.0-hr warm ischemia, and 1.0-hr warm ischemia and transplant $(P<0.05$ in all cases). (Right) BSP determinations between normal $(n=6)$ and 0.5 -hr warm ischemia $(n=4), 0.5$ - and 1.0-hr warm ischemia $(n=4)$, and $1.0 \mathrm{hr}$ warm ischemia and transplant $(n=6)$ were all statistically significantly different $(P<0.05)$.

\section{DISCUSSION}

${ }^{99 \mathrm{~m}} \mathrm{Tc}$-disofenin, a member of the iminodiacetic acid (HIDA) family of tracers, is actively taken up by hepatocytes via a mechanism similar to that involved in bilirubin uptake [13-15]. Previous attempts at measuring hepatic parenchymal transit were hampered by the fact that the liver is continuously and simultaneously extracting and excreting tracer [16]. This overlap of tracer uptake, transit, and excretion has made quantitative analysis quite difficult.
Deconvolutional analysis is a mathematical technique which permits "correction" of the hepatic time-activity curve for the changing blood concentration of tracer being presented to the liver. Continuous measurement of blood concentration of tracer (via the heart time-activity curve) allows recovery of the hepatic time-activity curve that would have occurred had the tracer been injected in the hepatic blood supply as a single short bolus without recirculation. This curve simulates a "single-pass" exposure of the liver to tracer and thus measurement of the time required for transit through the liver is readily calculated.

In this paper we validated that the deconvolution process used to reconstruct the hepatic time-activity curve from data acquired after intravenous injection accurately reflects the actual hepatic time-activity curve obtained from direct injection of tracer into the afferent blood supply of the liver. The mean transit time determined from the deconvolved curve and the "actual" curve correlated closely. The correlation between the deconvolved and actual hepatic time-activity curves was also found when the experimental animal had suffered unintended ischemic damage to the liver; both MTT determinations were similarly prolonged. These results are thus similar to our previous work in this area where it was shown that the deconvolution process accurately reconstructed renal time-activity curves generated

TABLE 2

HISTOLOGIC EXAMINATION OF LIVER BIOPSY SPECIMENS

\begin{tabular}{cccc}
\hline Group & Necrosis & $\begin{array}{c}\text { Vacuolar } \\
\text { changes }\end{array}$ & $\begin{array}{c}\text { Disorganization } \\
\text { of hepatic cords }\end{array}$ \\
\hline $\begin{array}{c}\text { Normal } \\
(n=4) \\
0.5 \text { hr of warm ischemia } \\
(n=5) \\
1.0 \text { hr of warm ischemia } \\
(n=4) \\
\begin{array}{c}\text { Transplant } \\
(n=6)\end{array}\end{array} \frac{1}{4}^{\frac{1}{5}}$ & $\frac{1}{4}$ & $\frac{1}{4}$ & $\frac{1}{5}$ \\
\hline
\end{tabular}


after the intravenous injection of ${ }^{131} \mathrm{I}$-orthoiodohippurate when compared to the renal time-activity curve obtained after direct injection of tracer into the renal artery of a uninephrectomized animal [6]. The correlation coefficient in that case was 0.97 . The technique of deconvolutional analysis appears to reliably provide a critical, previously missing element in the process required to quantitate organ function scintigraphically.

Using the reconstructed hepatic time-activity curves as a basis for quantitating hepatic function, we calculated the mean transit time (MTT), the time required in minutes for a molecule of bilirubin analog (disofenin) to enter and then exit the liver, under a variety of experimental conditions. The MTT increased progressively after increasing doses of ischemia/reperfusion in a pattern that paralleled results of simultaneously performed BSP determinations. It is possible that the distinction between the normal and $\frac{1}{2}-\mathrm{hr}$ ischemic groups could have been made on the basis of an effect of the surgical procedure itself rather than just ischemia since the normals had not undergone an abdominal incision with intraabdominal manipulation as the $\frac{1}{2}$-hr ischemic group had done. However, the $\frac{1}{2}-\mathrm{hr}$ ischemia, the $1-\mathrm{hr}$ ischemia, and the transplant group, all had undergone a similar abdominal incision and intraoperative manipulation. Thus the significant difference in MTT determination among these groups could not have been explained on the basis of an artifact related to the operative procedure itself. Results of MTT testing and BSP determinations correlated with the histologic evaluation only in that the transplanted group showed rather severe injury and this group also had the most prolonged MTT determinations and the most prolonged plasma $T_{1 / 2}$ for BSP. However, it was not possible to distinguish among the other experimental groups histologically as was the case with the scintigraphic and BSP testing techniques. We conclude from these results that the quantitative imaging with deconvolution is a relatively sensitive technique capable of identi- fying mild degrees of hepatic injury not associated with clear-cut histologic damage and that quantitative imaging with ${ }^{99 \mathrm{~m}}$ Tc-disofenin is as sensitive a technique as BSP testing in this regard.

Although in this experimental study MTT determinations and the $T_{1 / 2}$ of BSP were similarly prolonged after ischemic injury, in certain clinical situations the MTT determinations give more reliable information about hepatocyte function per se. If, for example, the clinical situation were one involving severe splanchnic vasoconstriction, proportionally less of the cardiac output would reach the liver and the liver would have less opportunity to extract the test substance from the blood, over time. In the case of the BSP determination the $T_{1 / 2}$ would be prolonged although the liver itself was functioning normally. The quantitative imaging technique with deconvolutional analysis would automatically take into account increased recirculation via the heart time-activity curve; the reconstructed hepatic time-activity curve would be unaltered; and, assuming normal hepatocyte function, the MTT determination would be normal. Thus, this quantitative imaging technique is essentially a flow-independent measurement of hepatocyte function, a feature which is a potential advantage in clinically sorting out problems related to hepatocyte injury versus changes in blood supply to the liver.

Quantitative imaging with deconvolution has the potential for wide clinical application. The technique is adaptable to the standard nuclear medicine minicomputer and provides rapid results, and injection of ${ }^{99 \mathrm{~m}} \mathrm{Tc}$-disofenin is not associated with the occasional severe reactions associated with BSP administration. An application which appeals to us particularly relates to liver transplantation. Quantitative scintigraphy could be used to evaluate the unstable potential liver donor or the immediate function of a questionable liver transplant in the perioperative period. Further studies, now being carried out, will test the application of this 
technology for the diagnosis of liver transplant rejection.

\section{REFERENCES}

1. Harvey, E., Loberg, M., and Cooper, M. Tc ${ }^{99 m}$ HIDA: A new radiopharmaceutical for hepatobiliary imaging. J. Nucl. Med. 16:533, 1975. (Abstract)

2. Rona, P. M., Baker, R. J., and Bellen, J. C. Technetium-99m pyridoxylideneglutamate: $A$ new hepatobiliary radiopharmaceutical. I. Experimental aspects. J. Nucl. Med. 16: 720, 1975.

3. Galli, G., Orlando, P., Massari, P., Bontazi, N., Magistrelli, P., and Loppola, R. ${ }^{99} \mathrm{~m}$ Tc-diethyl-IDA: The extraction efficiency of the liver. Eur. J. Nucl. Med. 8: 187, 1983.

4. Tarolo, G., Picozzi, R., Palagi, B., and Cammelli, F., Comparative quantitative evaluation of hepatic clearance of diethyl IDA and para butyl-IDA in jaundiced and non jaundiced patients. Eur. J. Nucl. Med. 6: 539, 1981.

5. Juni, J. E., Thrall, J., Froelich, J., Hichwa, R., and Clinthorne, N. A simple technique for reducing deconvolution artifact in scintigraphic studies. Proceedings, Med. Comp. First IEEE Computer Society Int. Conference on Med. Comp. Science/Computational Medicine. 1982. Vol. 4, 174.

6. Juni, J. E., Thrall, J., Froelich, J., Wiggins, R., Campbell, D. A., and Tuscan, M. A new technique for deconvolutional analysis method and validation. J. Nucl. Med. In press.

7. Juni, J. E. Differentiation of obstructive and nonobstructive jaundice by deconvolutional analysis. Submitted for publication.
8. Sherlock, S. Diseases of the Liver and Biliary Systems, 7th ed. Oxford: Blackwell, 1985.

9. Cornelius, C. E. Liver function. In J. J. Kaneko (Ed.), Clinical Biochemistry of Domestic Animals, 2nd ed. New York: Academic Press, 1970. pp. 161-230.

10. Calne, R. Y. Technique in the pig. In R. Y. Calne (Ed.), Liver Transplantation. New York: Grune and Stratton, 1983. pp. 9-16.

11. Lelange, J. J., Hoitsma, H. F., and Meijer, S. Anesthetic management in experimental orthotopic liver transplantation in the pig. Eur. Surg. Res. 16: 360, 1984.

12. Marino, I. R., and LeLuca, G. Orthotopic liver transplantation in pigs. An evaluation and different methods of avoiding the revascularization syndrome. Transplantation 40: 494, 1985.

13. Harvey, E., Loberg, M., Ryan, J., Sikowski, S., Faith, W., and Cooper, M. Hepatic clearance mechanism of TC-99m-HIDA and its effect on quantitation of hepatobiliary function. J. Nucl. Med. 20: $310,1979$.

14. Javitt, N. B. Hepatic bile formation II. N. Engl. I. Med. 295: 1511, 1976.

15. Vallabhajosula, S., Nunes, R., Okuda, H., Strashun, A., Goldsmith, S. J., and Berk, P. D. Studies in the mechanism of hepatocellular uptake of ${ }^{99 \mathrm{~m}} \mathrm{Tc}$-DISIDA. J. Nucl. Med. 27: 938, 1986.

16. DiJorge, M. W. C., Pauwels, E., Hennis, P., Ruiter, D., Koch, C., Blom, J., Schalim, S., and Terpstra, J. Cholescintigraphy with ${ }^{99 \mathrm{~m}} \mathrm{Tc}$-Diethyl-IDA for the detection of rejection of auxillary liver transplants in pigs. Eur. J. Nucl. Med. 8: 485, 1983. 